\title{
Teratogenetische Studien.
}

\author{
Von \\ Prof. P. Mitrophanow.
}

(Aus dem Zootomischen Institut der Universität Warschau.)

Mit Tafel XIII-XV and 13 Figuren im Text.

Eingegangen am 3. December 1894.

I.

Unter einigen Hunderten Keimscheiben des Hühnchens, welche im Laufe der letzten Jahre im Zootomischen Laboratorium in Folge des beständig wirkenden Inkubators erhalten wurden, bemerkte man auch eine kleine Anzahl ziemlich seltener und theoretisch interessanter Missbildungen. Letztere erhielt man auch manchmal bei angenscheinlich normalen Bedingungen, aber öfters konnten sie durch eine absichtliche Veränderung der Entwickelungsbedingungen hervorgerufen werden.

So hat man unter Anderem, in Übereinstimmung mit CAMILLE Dareste (1, pag. 118), bemerkt, dass bei einer Erniedrigung der Temperatur $\left(32^{\circ}-34^{\circ} \mathrm{C}\right.$.) nicht nur die Entwickelung sich verspätet, sondern auch oft scharf ausgedrückte Abweichungen in der Entwickelung des Primitivstreifens vorkommen, Abweichungen, welche vom Standpunkte der genetischen Beziehungen sehr interessant sind.

Ofters verändert sich dabei das hintere Ende des Primitivstreifens, indem es Erweiterungen, Seitenvorsprünge und sogar Zerspaltungen bildet. Letztere Erscheinungen haben für uns jetzt einen besonderen Werth.

Man bekommt sehr eigenthümliche Abweichungen bei der Bebrïtung der Eier, deren eine Hälfte (des Schwanzes oder des Kopfes) mit Lack oder Kollodium bedeckt ist. Die Veränderung der normalen Bedingungen besteht in diesem Falle in der Versperrung oder wenigstens in dem Hindernisse des freien Zuganges der Luft von der einen Seite. Die Experimente in dieser Beziehung 
sind erst angefangen, und man muss mehr Material haben, um Schlussfolgerungen bestimmter Art zu machen 1).

Allem Anscheine nach sind dagegen von der ausschließlichen und direkten Einwirkung der Veränderung der äußeren Bedingungen jene Abweichungen unabhängig, welche zur Bildung zweier oder mehr Embryonen in einem Eie führen, aus welchen später Doppelund Mehrfachmissbildungen herstammen.

In der Litteratur ist verhältnismäßig nur eine geringe Anzahl solcher Beobachtungen angegeben; und im gegenwärtigen Falle denke ich solche zu beschreiben, welche unter den erwähnten Verhältnissen in meine Hand gekommen sind.

\section{Die Keimscheibe mit zwei Primitivstreifen und dem dritten scheinbaren).}

(Taf. XIII Fig. 1; Textfigur 1.)

Dieses Präparat hat vor zwei Jahren Herr Ersmond erhalten, als er sich mit dem Erreichen von Veränderungen des Primitivstreifens

Fig. 1.

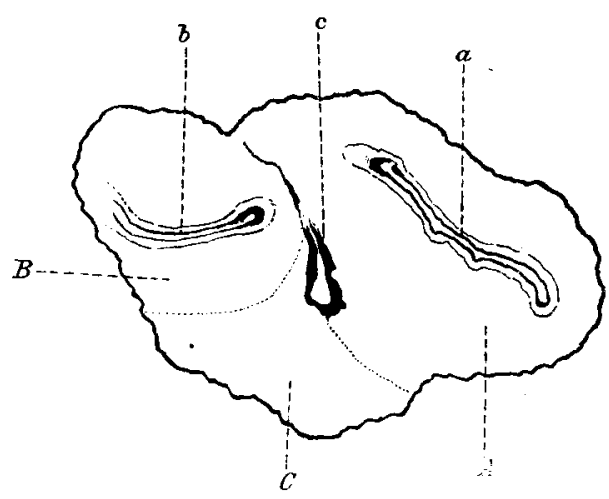

Keimscheibe des Hühnchens nach einem Photogramm. $a$ und $b$ Primitivstreifen. $c$ scheinbarer Primitivstreifen von Dotterresten gebildet. $A, B$ Area pellucidae beider Anlagen. $C$ zwischenliegendes Feld ohne Primitivstreifen. bei einer erniedrigten Temperatur beschäftigte. $\mathrm{Er}$ hat dasselbe liebenswürdig zu meiner Verfugung gegeben.

Die angegebene Definition der Keimscheibe fordert eine Erläuterung, da die Natur des sogenannten dritten Primitivstreifens nicht ganz klar ist. Bei schwacherVergrößerung und auf Photographien (Taf. XIII Fig. 1) können alle drei Streifen ohne großen Zweifel für primitive angenommen werden, bei näherer Beobachtung sind aber nur die zwei an den Seiten gelegenen in ihrem Charakter einander und den normalen Primitivstreifen von entsprechender Entwickelung ähnlich, der dritte

1: Wir finden die Geschichte dieser Methode bei Camille Dareste $\left(\mathbf{1}, 1^{\mathrm{e}} \mathrm{p} . \mathrm{Ch} . \mathrm{V}, \S 1\right)$. 
(mittlere) ist aber von ihnen ganz verschieden, obgleich in einigen anormalen Fällen der Primitivstreifen eben so aussehen kann.

Der größere Primitivstreifen (Textfigur 1 a), welcher $2 \mathrm{~mm}$ lang und auf der Photographie, welche von der unteren Seite abgenommen war, der rechte bei der normalen Lage der Keimscheibe ist, zeigt bestimmt, wo der Kopf der Missbildung sein müsste; an seinem einen Ende beobachtet man eine eben solche Verdickung und eine Anhäufung der Zellen, welche an dem vorderen Ende des normalen Streifens vorkommt; folglich ist es das Kopfende. Der linke Streifen $(b)$, welcher fast doppelt so kurz wie der rechte ist $(1,25 \mathrm{~mm}$ lang), hat eine eben solche Verdickung am Kopfende. Beide Streifen nähern sich einander vermittels ihrer Kopfenden und beide sind gekrümmt: der linke bildet eine bogenförmige Krümmung, der rechte bildet deren zwei, in der Mitte und am Ende des Schwanzes; außerdem sind seine Umrisse unregelmäßig und stellenweise, besonders am hinteren Ende, bilden sie eine Art von Seitenvorsprïngen. Bei der Beobachtung von der äußeren Oberfläche und bei einer bedeutenden Vergrößerung zeigt das vordere Ende jedes Streifens eine Erweiterung und eine Vertiefung, welche von cylinderförmigen Zellen klar begrenzt sind. Am hinteren Ende sind solche Zellen in den Wänden des Streifens nicht so dicht und nicht so regelmäßig gruppirt, wodurch auch die erwähnte Unregelmäßigkeit in den Umrissen des größeren Streifens erklärt wird, welcher dabei auch am hinteren Ende mehr erweitert ist. Das vordere Ende des rechten Streifens zeigt schon die Differenzirung $(c h)$ der Chordaanlage, wenn auch anf unansehnlicher Strecke.

Auf der Grenze zwischen beiden Streifen, näher und perpendikulär zu dem linken, beobachtet man quasi einen dritten Primitivstreifen $(c)$. Bei einer aufmerksamen Forschung kommt man jedoch zu dem Schlusse, dass es eigentlich mit dem Primitivstreifen nichts Gemeinsames hat. Die äußere Schicht des Ektoderms dehnt sich uiber dieser Bildung ohne jegliche Veränderungen aus und zeichnet sich gar nicht von den Rändern der Area pellucida aus.

In Wirklichkeit kommen ja hier die Ränder beider Areae pellucidae zusammen, deren Primitivstreifen oben beschrieben worden sind. Die Figur des Primitivstreifens wird ja durch eigenthümlich gelegene Partikeln des Dotters gebildet, welches im Falle der isolirten Lage der zusammentretenden Anlagen der Doppelbildung. hier den Keimwall gebildet bätte. 
Auf diese Weise wird das dem ersten Anscheine nach zusammengesetzte Bild des dreifachen Keimes vereinfacht und zeigt die ungleich entwickelten Keime zweier Embryonen, welche in ihrer späteren Entwickelung augenscheinlich durch ihre vorderen Enden zusammengewachsen wären und, wie man mit großer Wahrscheinlichkeit annehmen kann, einen dem Fall ähnlichen gebildet hätten, dessen Beschreibung unmittelbar nach diesem folgt.

In der Litteratur hat man sehr wenig Beispiele einer in ihrer Bildung so frihen doppelten Missbildung. Bei Gerlach (2, Taf. III Fig. 1) haben wir einen ähnlichen Fall für ein Hühnchen nach zwanzigstündiger Bebritung. „Somit waren in einer gemeinsamen Area pellucida zwei Primitivstreifen vorhanden und es handelte sich um eine sehr frühe Doppelbildung, wodurch sich auch die retardirte Entwickelung der Embryonalanlagen erklärte .... Der hintere Primitivstreifen besaß eine Länge von $1,8 \mathrm{~mm}$ und war entschieden besser entwickelt als der linke vordere, der nur 1,6 mm lang war. Mit ihren centralen oder Kopfenden waren die beiden Streifen in das Embryonalschild hinein vorgedrungen, welches von der dunkleren Randzone des durchsichtigen Fruchthofes sich nur schwach abhebt. Die Entfernung zwischen den Kopfenden der beiden Streifen beträgt $0,4 \mathrm{~mm}$ " (2, pag. 29).

Ein anderer Fall der Keimseheibe mit zwei Primitivstreifen ist bei Klaussner angeführt (3, pag. 50, Taf. X Fig. 60). »Von der Schmalseite her strahlt links und rechts je eine Embryonalanlage gegen die Mitte des Fruchthofes ein. Die linkerseits gelegene beginnt mit mäßig breiter Anlage dicht am Rande der Area pellucida, von wo aus der Primitivstreifen in gestreckter Richtung dem Centrum zu verläuft. Der Kopffortsatz ist kaum angedentet. Der rechtseitige zeigt eine scharf kontourirte Sichel, die in einiger Entfernung von der Grenze des hellen Fruchthofes liegt. Der Primitivstreif strebt, wie linkerseits, gegen die Mitte des Fruchthofes zu. Von einem Kopffortsatz ist hier nichts zu sehen. Beide Kopftheile sind einander bis auf geringe Distanz genähert, beruhren sich jedoch nicht. Die beiden Anlagen stehen in einem Winkel von $160^{\circ}$ zu einander. *

Den dritten Fall finden wir in BurckhardT's Mittheilung (4) In der Mitte der Keimscheibe liegt eine einfache Area pellncida von rautenförniger Gestalt mit abgehenden Ecken. Der langen Achse dieser letzten entlang liegen zwei Primitivstreifen, welche mit ihren Kopfenden zu einander gekehrt und nur durch eine lichte 
Querzone von einander geschieden sind. Beide sind auf derselben Entwickelungsstufe, aber augenscheinlich etwas zurïckgeblieben, da das Ei 19 Stunden bebrïtet wurde und keiner von den beiden die geringste Spur von dem Kopffortsatze enthält. Die äußeren Umrisse der Streifen sind schwach und die Furchen sind nur in den Kopf- und Schwanzenden sichtbar, in der Mitte jedoch unterbrochen (l. e. pag. 432, der Holzschnitt im Text).

\section{Der doppelte Embryo des Hühnchens mit einem Kopfe.}

(Taf. XIII Fig. 2.)

Diesen Fall hat man noch vor drei Jahren bei ungefaihr normalen Bedingungen der Bebritung bekommen.

Beide Embryonen sind fast auf derselben Entwickelungsstufe, obgleich der rechte $(2,75 \mathrm{~mm}$ lang) etwas kürzer als der linke $(3 \mathrm{~mm}$ lang) ist.

Die Verschiedenheit in der Länge hängt hauptsächlich davon ab, dass letzterer auf einer größeren Ausdehnung den Primitivstreifen behalten hat (von $1,33 \mathrm{~mm}$, während der rechte nur von $1,25 \mathrm{~mm}$ ist).

Die Areae pellucidae beider Embryonen sind auf derselben Linie gelegen und bilden einen hellen Streifen, welchem entlang die mit ihren Köpfen zusammengewachsenen Zwillinge gelegen sind. Nur von einem Rande in der Mitte, in der Kopfgegend, bildet diese gemeinsame Area pellucida einen dreieckigen Vorsprung. Die gemeinsame Area vaseulosa ist verhältnismäßig wenig entwickelt und breiter bei dem linken Embryo.

. Die Anzahl der klar ausgedrükten Somiten bei dem linken Zwillinge ist sieben, bei dem rechten sechs. Das Nervenrohr ist bei dem ersten selbständig vom Mittelhirne an, wo es soeben anfängt sich zu schließen, bei dem zweiten ist es selbständig nur vom Hinterhirne an, in dessen Bezirk es noch ganz offen ist.

Das Zusammenfließen beider Zwillinge ist eben hauptsächlich ausgedrickt dureh die Vereinigung der Vordertheile ihrer Gehirne und durch die Bildung um den gemeinsamen Kopftheil der gemeinsamen Amnionfalte, welche aus dem Zusammenfließen beider Amnionfalten herstammt, dessen Spuren auf der Photographie noch sichtbar sind.

Dem Gesagten gemäß sind in den gemeinsamen Kopftheil vom linken Zwillinge das ganze Vorderhirn, vom rechten gleichfalls das Vorderhirn, so viel es hier für ansgebildet angenommen werden 
kann, und der größte Theil des Mittelhirns gekommen. Überhaupt ist das vordere Ende dieses Zwillings mehr missgestaltet und sein Hirn ist im Gebiete des Mittelhirns gekrïmmt.

Es ist schwer, bestimmt zu sagen, was sich durch Zusammenfließen beider Zwillinge gebildet hat: im gemeinsamen Hirn, welches die unmittelbare Fortsetzung des Mittelhirns des linken Zwillings bildet und zu welchem sich die Erweiterung des Hirns des rechten anschließt, kann man gar keine bestimmten Bildungen beobachten, obgleich das Präparat von beiden Seiten bei ansehnlichen Vergrößerungen studirt worden ist ${ }^{1}$ ).

Die hier beschriebenen Zwillinge sind nicht ganz selten. So finden wir bei Rauber (5a, Taf. VIII Fig. 34) fast denselben Fall, nur auf einer etwas späteren Entwickelungsstufe. Ähnliche doppelte Missbildungen sind auch bei Camille Dareste dargestellt (1, Pl, XV fig. 1 et 2; fig. 59, pag. 517). Bei Gerlach (2) sind ähnliche Fälle aus Rauber (Taf. IV Fig. 5), von Baer (Fig. 7), Dönitz (Fig. 8), Ahlfeld (Taf. V Fig. 4) und Tompson (Taf. VI Fig. 5) angeführt. Ihnen nähern sich auch die Fälle auf den Fig. 61-63, Taf. XI und XII, welche KLaussner (3) bei dem Hühnchen beschrieben hat, obgleich sie alle solche Zwillinge darstellen, welche noch nicht zusammengewachsen sind.

\section{Die Keimscheibe mit den Anlagen einiger Primitivstreifen.}

(Taf. XIII Fig. 3; Taf. XIV Fig. 7 und 8.)

Diese Keimscheibe stellt äußerst merkwürdige Abweichungen dar und ist von mir in der letzteren Zeit, das heißt, aus den Herbsteiern, unter ganz anormalen Bedingungen der Entwickelung, erhalten worden.

Erstens war die Temperatur im Inkubator bis $32^{\circ} \mathrm{C}$. erniedrigt, nur Abends stieg sie bis $34^{\circ} \mathrm{C}$. Das war in der Absicht geschehen, die Entwickelung zuriickzuhalten, wobei Missbildungen öfter vor* kommen. Zweitens war die Hälfte des Eies nach der der Länge nach laufenden Achse vermittels einer dünnen Kollodiumschicht zugedeckt, um den Eintritt der Luft zu verhindern, und das Ei war

1) Die beiden eben beschriebenen Missbildungen sind nur in toto studirt worden, da man die Präparate für die Sammlung des Zootomischen Kabinetts behalten hat. Die Keimscheiben sind in einer Glaszelle zwischen zwei Deckgläschen in Kanadabalsam eingeschlossen, damit es möglich sei, sie von beiden Seiten mit bedeutenden Vergrößerungen zu beobachten. Diese Zelle ist zwischen zwei Kartonplatten mit runden Löchern eingeklebt. 
in den Inkubator so gelegt, dass die gedeckte Hälfte des Eies das Schwanzende des Embryos einschloss, folglich auf solche Weise, dass, als das Ei mit seinem spitzen Ende nach rechts gelegen war, die gedeckte Hälfte nach der Seite des Beobachters gekehrt war, und dass die Grenzlinie zwischen der lackirten und der freien Oberfläche sich in einer senkrechten Fläche befand.

Indem ich hier diese Bedingungen mittheile, will ich gar nicht damit sagen, dass dieselben unmittelbar die Missbildung verursacht haben, welche gleich beschrieben wird. Ihr Einfluss ist unstreitig, aber die Grundursache der anormalen Entwickelung muss in diesem Fall bedeutend auf die Eigenthümlichkeiten der Organisation des Eies selbst zurückgeführt werden.

Die Keimscheibe ist am dritten oder vierten Tage der Bebriitung abgenommen worden. Die Zeit der Bebrutung hat in diesem Fall keine große Bedeutung, da bei den anormalen Bedingungen die Entwickelung sich sehr versehiedenartig verspätet. Es ist nur wichtig in Betracht zu ziehen, dass die Missbildung, von der wir sprechen werden, augenscheinlich von langer Daner war.

Die Area pellucida stellt Umrisse eines unregelmäßigen Kreises dar, von rechts ist dieser Kreis unregelmäßig abgeschnitten (Taf. XIV Fig. 7). Nach der Entwickelung des Primitivstreifens beurtheilend, entspricht die Keimscheibe einer 15-16 stündigen normalen Bebrütung, obgleich sie am dritten oder vierten Tage abgenommen worden ist. Auf der Area pellucida unterscheidet sich vor Allem in ihrem unteren Theile eine trapezähnliche Verdickung (h), deren Ecken sich in kleine gekrümmte Furchen ( $a$ und $b$ ) ausdehnen, welche von oben unzweifelhafte, in verschiedenem Grade gekrümmte und in einem Winkel zu einander liegende Primitivstreifen darstellen. Die Entfernung zwischen ihren oberen Enden beträgt $1 \mathrm{~mm}$.

Als Streifen sind sie klar ausgedrückt nur im Vordertheil; im mittleren Theil richten sich ihre inwendigen Ränder zu einander und scheinen zusammenzufließen; in Folge dessen sind die Umrisse des oben erwähnten Trapez entstanden.

Die hinteren Enden beider Primitivstreifen setzen sich in die hinteren Ecken von diesem Trapez fort, welches auf diese Weise und auf einer bedeutenden Strecke eine fur beide hier beschriebene Streifen gemeinsame Verdickung bildet. Der ganze hintere Theil der Keime hat noch im Gebiet der Area pellucida scharfe Grenzen, welche von der lokalen Verdickung verursacht worden sind $(k)$.

Das Interesse dieser Keimscheibe besteht jedoch darin, dass 
zwischen den vorderen Enden der beiden erwähnten Primitivstreifen sich noch drei befinden; zwei davon sind mit einander verbunden $(c$ und $d)$, der dritte $(e)$ ist frei. Die Entfernung zwischen den unteren Enden der ersten zwei Primitivstreifen beträgt $0,3 \mathrm{~mm}$. Es ist eine Frage, in wie fern dieselben den Primitivstreifen entsprechen.

Außerdem beobachtet man auch neben dem rechten Rande der oberen Theile der Area pellucida eine kleine aberrante doppelte Verdickung $(f)$, welche mit Hilfe cylinderförmiger Zellen gebildet wird, wie auch die soeben erwähnten sekundären Streifen. Die Natur aller dieser Bildungen kann nur auf Schnitten erläutert werden.

In dieser Absicht wurde die Keimscheibe nach vielseitigem Studiren in toto und nachdem sie photographirt worden war, in der auf Taf. XIV Fig. 7 bezeichneten Richtung geschnitten ${ }^{1)}$.

Wenn wir diese Schnitte, vom oberen Ende der Keimscheibe an, studiren, überzeugen wir uns vor Allem davon, dass die bezeichnete aberrante Verdickung $(f)$ eine wirkliche Furche ist und dabei keine einfache, sondern eine doppelte, wie es auf der Abbildung in toto sichtbar ist (Taf. XIV Fig. $7 f$ und $g$ ). Das Ektoderm ist im Gebiet dieser Furchen sehr bedeutend verdickt (Taf. XIV Fig. 8, $I I$ ), bis $40 \mu$, während das umgebende Ektoderm nur $7,5 \mu$ und am Rande der Area pellucida 6 , beträgt, und wenn die linke Furche als eine einfache asymmetrische Vertiefung und eine Falte ohne tiefere Differenzirung erscheint (Taf. XIV Fig. 8, $I, I I f$ ), so stellt die rechte, ohne so tief zu sein, die Differenzirung der Elemente auf ihrem Boden (Taf. XIV Fig. 8, II $g$ ) in der gleichen Art dar, wie man es auf dem Boden des wirklichen Primitivstreifens beobachtet.

Da das Entoderm hier ganz abgesondert ist und seinen speciellen Charakter hat, so könnte man hier von der Bildung des mittleren Keimblattes sprechen.

Die linke Falte beginnt viel höher als die rechte (Taf. XIV Fig. 7), ist in ihrem oberen Theil symmetrisch und erinnert auch an einen kleinen, nicht tiefen Primitivstreifen (Taf. XIV Fig. 8, $I, f$ ), welcher hier itbrigens gar keine Beziehungen weder zum Mesoderm noch zum Entoderm hat.

1. Die Dicke der Schnitte beträgt $1 / 1.50 \mathrm{~mm}$. Vorläufige Bearbeitung --. 30/0ige Salpetersäure und eine allmähliche Herüberführung der Keimscheibe in Alkohol. Färbung - Spirituslösung des Hïmatoxylins mit Chlorkalcium und Alaun. 
Letzteres besitzt in der ganzen oberen Hälfte der Keimscheibe, besonders in ihrem mittleren Theil (Taf. XIV Fig. $7 i$ ), wo es auch in toto durchseheint, eine bedeutende Dicke und zeichnet sich durch denselben schäumenden Charakter aus, welcher ihm uberall in der Nähe des Keimwalles eigen ist.

Die erwähnten Falten haben eine sehr kleine Ausdehnung und ihnen folgen einige Schnitte, auf welchen das Ektoderm in seinem mittleren Theil nur leicht verdickt ist. Danach aber wird diese Verdickung rechts größer, und da erscheint wieder eine Vertiefung, welche nach der Richtung der Schnitte der kleinen Furche entspricht (Taf. XIV Fig. 7 e), da ihr oberes Ende sich am meisten nach oben hebt. Der Boden dieser kleinen Furche zeichnet sich im Vergleich mit dem nächsten Ektoderm durch keine besonderen Veränderungen aus, obgleich die Lage ihrer Elemente klar darauf deutet, dass sie nicht das Resultat einer zufälligen mechanischen Bildung der Falten in der Keimscheibe, sondern die direkte Folge der Erscheinungen des Wachsthums in diesem Gebiet ist.

Dasselbe kann man auch von den kleinen Furchen $d$ und $c$ sagen, welche auf den folgenden Schnitten erscheinen, und auf der ganzen Strecke aller drei Furchen $c, d$ und $e$ bleiben sich die Beziehungen gleich. Die Furchen können mehr oder weniger tief sein (am tiefsten im mittleren Theil), aber tiberall erscheinen sie als der Ausdruck einfacher ektodermischer Falten des verdickten Ektoderms, wie auch die oben beschriebene aberrante Falte $f$.

Nicht nur die Anordnung der Zellenelemente dieser Falten spricht für ihre natürliche Herkunft und nicht für eine solche, welche durch Bearbeitung und die nach dem Tode stattgefundenen mechanischen Bedingungen hervorgerufen worden wäre; dafür spricht auch die Beziehung zu jenen des Entoderms und die Beschaffenheit der zwei anderen Furchen $a$ und $b$, zu deren Beschreibung wir jetzt übergehen.

Schon bei der Beobachtung der Keimscheibe in toto machten diese Furchen den Eindruck wirklicher Primitivstreifen. Auf den Schnitten wird das ganz augenscheinlich bestätigt, wie es oben auch für die Furche $g$ gezeigt worden ist. Der von uns angenommenen Ordnung des Studirens der Schnitte gemäß erscheint darauf zuerst das obere Ende der Furche $b$.

Auf der Taf. XIV Fig. 8, III ist der Schnitt ungefähr durch das obere Drittel dieser Furche dargestellt. Da derselbe Schnitt auch durch das hintere Ende der Falte $e$ gegangen ist, so ist ihre Furche 
schwach ausgedrückt, während die Furchen $c$ und $d$ es sehr deutlieh sind.

Die erste $(c)$ zeichnet sich durch einen eben solchen asymmetrischen Charakter aus, wie die beschriebene Falte $f$; die zweite $(d)$ nähert sich mehr ihrem äußeren Aussehen nach dem typischen Primitivstreifen. (Die Falte $e$ ist auch in ihrem mittleren Theil asymmetrisch, aber nach einer anderen Seite hin, nämlich nach der des Primitivstreifens $b$.)

Endlich befindet sich auf demselben Schnitt nur das ganz oberè Ende des Primitivstreifens $a$, desshalb ist derselbe hier äußerst wenig ausgedrückt und bietet hier noch nicht die dafür typische Differenzirung.

Obgleich auf dieser Zeichnung die Größe nicht genügend ist, um Alles ausführlich zu erklären, ist die Differenzirung auf dem Boden der Furche $b$ vollständig klar. Ihre verdickten (bis $47, a$ ) Wände theilen die Zellenelemente in beiden Richtungen und besonders in derjenigen der Falte $d$ ab.

Einzeln sind diese Elemente unter allen drei Falten $c, d$ und $e$ sichtbar, sind aber uiberall von ihnen unabhängig, während in den Wänden der Furche $b$, eben so wie in dem mittleren und unteren Theil der Furche $a$ (Taf. XIV Fig. 7; 8, III, IV; Taf. XIII Fig. 3) neben den Zellen, welche sich absondern und locker gelegen sind, man noch zahlreiche karyokinetische Figuren beobachtet. Der Primitivstreifen $a$ ist noch typischer.

Die Schnitte, welche durch das hintere Ende des Primitivstreifens $a$ gehen, greifen schon weder die oben beschriebenen Falten, noch die Furche $b$ mit und gehen durch diejenige trapezähnliche Verdickung, welche noch früher bei dem Studiren der Keimscheibe in toto (Taf. XIV Fig. $7 h$ ) bemerkt worden ist und deren Umrisse durch die aus einander gehenden Ränder der Furchen $a$ und $b$ bestimmt werden (Taf. XIII Fig. 3).

Diese Verdickung ist auf den Schnitten in der Hinsicht beachtenswerth, dass ihr verdicktes Ektoderm denselben Charakter hat, wie der Boden des Primitivstreifens und, gleich dem letzteren, zahlreiche Zellenelemente abscheidet, welche den ganzen Raum bis zur dünnen und hier schwach abgesonderten entodermischen Platte ausfillen (Taf. XIV Fig. 8, IV).

Denselben Charakter behält sie auch hinter dem hinteren Ende der Furche $a$, in ihrem ganzen Gebiet.

Auf diese Weise scheint die trapezihnliche Verdickung (mit dem 
Entoderm bis $75 "$, dick) nach ihrer Herkunft, als das Resultat des Zusammenfließens zweier Primitivstreifen $a$ und $b$, und nach ihrer histologischen Struktur, nichts Anderes zu sein als ein erweiterter kleiner Primitivstreifen mit einer unansehnlichen Vertiefnng in der Mitte (Taf. XIV Fig. 8, $V, h$ ).

Aus dem Gesagten ist es klar, dass die Keimscheibe, welche wir beschreiben, eine sehr zusammengesetzte Bildung ist. Vor Allem sondern sich darin die unzweifelhaften Keime der drei Primitivstreifen $a b$, von denen einer $(g)$ ganz einzeln liegt, so klein ist und eine so ausschließliche Lage dem Rande der Area pellucida entlang einnimmt, dass man ihn Anfangs gar nicht beachtet hat. Die zwei anderen Primitivstreifen gehen vermittels ihrer oberen und augenscheinlich Kopfenden neben dem Centrum der Area pellucida ans einander, hinten aber nähern sie sich einander und bilden eine Art von gemeinsamem erweitertem Primitivstreifen (h).

Eine solche Lage dieser beiden Primitivstreifen stellt ein sehr frühes Stadium des doppelten Keimes des zukïnftigen Embryos mit zwei Köpfen, mehr oder weniger getheilten Körpern und einem gemeinsamen Schwanztheil dar. Wir werden später eben mit einem solchen Zwillingspaar zu thun haben.

In welcher Beziehung zu diesen zusammengewachsenen Primitivstreifen der erwähnte dritte $(g)$ steht, ist schwer zu sagen; wir haben auch keinen Anhalt, um zu erkennen, wo letzterer das Kopfund wo er das Schwanzende hat. Was die vier Falten $(c, d, e, f)$ oder ssekundären Furchen ", wie ich sie oben genannt habe, betrifft, so kann man sie, der mikroskopischen Analyse auf den Schnitten gemäß, nicht für wirkliche Primitivstreifen annehmen, man kann sie aber auch nicht als zufällige Bildungen betrachten, welche von der Bearbeitung oder von anderen Ursachen nach dem Tode der Keimscheibe herstammen. Alle sind wirkliche Falten, welehe während des Wachsthums der Keimscheibe erschienen sind, als das Resultat seiner anormalen Richtung, welche ihrerseits in bedeutendem Grade durch die unnatürlichen äußeren Bedingungen hervorgerufen worden ist.

Der Einfluss der Faktoren, welche die Entwickelung zurtuckhalten.

Erinnern wir uns daran, dass die Entwickelung dieses Eies bei einer sehr erniedrigten Temperatur von $32^{\circ} \mathrm{C}$. stattfand. Der zurkickhaltende Einfluss der erniedrigte Temperatur war noch durch 
die halbe Lackirung des Eies erhöht. Das Alles stellte das Ei, welches selbst vielleicht schon inwendig anormal war, in solche Bedingungen, wobei die Erscheinungen des primären Wachsthums ganz gut stattfinden konnten, wobei aber die weiteren Komplikationen der Organisation keinen genügenden Impuls zu ihrem Erscheinen hatten. Es gab eine vollständige Möglichkeit für die Bildung des Blastoderms und seine ersten Veränderungen, aber die Bedingungen für das Erscheinen der Nervenplatte, der Medullarwülste, der Nervenfurche etc. fanden nicht statt. Die allgemeine Intensität der Lebensprocesse, welche in der Keimscheibe vorkommen, war dafür ungeniügend.

Während aber bei normalen Bedingungen die ersten Entwickelungsprocesse ihrer Zeit durch fernere Komplikationen der Organisation ersetzt werden, hat sich in diesem Fall bei zurückgehaltener Entwickelung die Bildung des Blastoderms, welche ihre normalen Grenzen erreicht hat und zur rechten Zeit nicht durch fernere Komplikationen ersetzt worden ist, sich auf einen größeren Zeitraum (ungefähr 3 Tage) ausgedehnt und in Folge dessen eine größere Entwickelung bekommen. Das Blastoderm erschien hypertrophirt, aber nicht in dem Sinne, dass es einen größeren Raum eingenommen hätte, - im Gegentheil, die Area pellucida war verhältnismäßig klein, sondern in dem, dass die Anzahl seiner Elemente bedeutend zugewachsen war, und in Folge dessen sein mittlerer Theil erstens viel dicker als der normale wurde, und zweitens derselbe sich entweder als Blase heben oder die Falte bilden musste, da er eine größere Oberfläche einzunehmen strebte. Letzteres geschah in unserem Fall. Die Falten $e$ und $f$ sind einfache abgesonderte sackartige Vertiefungen, während die Falten $c$ und $d$ als Theile einer gemeinsamen Falte erscheinen, wie man es bei einer stärkeren Vergrößerung sieht (Taf. XIII Fig. 3). Sogar die Umrisse ihrer oberen Enden haben hier den Charakter von Vertiefungen behalten.

Auf diese Weise ist ihre Herkunft vollständig mit den Erscheinungen des Wachsthums verbunden und fand noch beim Leben der Keimscheibe statt, welche trotz der langen Bebriitung eine so frühe Entwickelungsstufe behalten hat. Und dass die Keimscheibe im Moment ihrer Fixirung lebte, wird durch die zahlreichen karyokinetischen Figuren und durch den guten Zustand ihrer Elemente bestätigt.

Das zusammengesetzte Bild der beschriebenen Keimscheibe 
bietet folglich Erscheinungen zweier Arten; erstens das Erscheinen von drei Anlagen der Primitivstreifen, zweitens dasjenige von drei Falten, welche gar keine Beziehung zu der normalen Entwickelung haben und als Resultat der vorwiegenden Entwickelung der primitiven Processe auf begrenztem Raum erscheinen.

In der Litteratur finden wir keine entsprechenden Fälle einer so frühen und so komplicirten Missbildung.

\section{Ein zweiköpfiger Embryo des Hühnchens.}

(Taf. XIV Fig. 9.)

Diese Doppelbildung war bei ziemlich normalen Bedingungen erhalten 1$)$.

Die Area pellucida dieser Doppelbildung hat biscuitartige und dabei hinsichtlich ihrer langen Achse nicht ganz symmetrische Umrisse. Ihr entlang sind die beiden Embryonenkörper gelegen, welche vermittels ihrer Mittel- und Hintertheile mit einander zusammengeflossen sind, ungefähr auf der Strecke zweier Drittel der ganzen Länge (Taf. XIV Fig. 9).

Bei deren Anblick fällt zuerst auf, dass einer von den Embryonen $(A)$ verhältnismäßig größer und, nach der Entwickelung seiner Organe beurtheilend, älter als der zweite $(B)$ ist. Die Länge des ersten beträgt vom Vorderende des Köpfehens bis zum Schwanzende $5 \mathrm{~mm}$, die des zweiten $4 \mathrm{~mm}$. Die langen Achsen der beiden Embryonen treffen im mittleren und unteren Drittel mit der Achse der Area pellucida zusammen, aber von dem vorderen Drittel an gehen ihre Achsen in einen Winkel von ungefähr $45^{\circ}$ nach rechts und links aus einander, dabei so, dass die Achse des ersten Embryos $(A)$ fast gar nicht auf irgend eine Seite abweicht; letzterer stellt so zu sagen einen Stamm vor, von welchem in Form eines Seitenastes der zweite geht $(\boldsymbol{B})$. Eine solche untergeordnete Lage und verhältnismäßig kleinere Größe des zweiten geben ihm den Charakter eines Parasiten.

Nach der Entwickelung der verschiedenen Organe kann man das Alter des hier beschriebenen ersten Embryos $(A)$ durch den

1) Die äaßere Beschreibung dieses Exemplars, ebenso wie die Bildung bei ihm des peripherischen Nervensystems, war in der III. Lieferung der Arbeiten aus dem Zootomischen Laboratorium der Universität Warschane 1892 von A. Ptizin (6), welcher unter meiner Leitung gearbeitet hat, verfasst. Davon stammen meine weiteren, diese Gegenstände betreffenden Auseinandersetzungen, sowie die Figuren 9, 12-16, Taf. XIV. 
Anfang des zweiten Tages der Bebritung (ungefähr von 26 Stunden nach Duval's Angaben und zwischen 30-33 Stunden bei einer Temperatur von $35^{\circ}$ C.) bestimmen. Es ist schwer, etwas Bestimmtes über das Alter des zweiten $(\boldsymbol{B})$ zu sagen, da seine unterworfene Lage wahrscheinlich die Ursache jener Unverhältnismäßigkeit und Ungleichmäßigkeit war, welche man in der Entwickelung seiner Organe beobachtet.

Ein solcher Fall doppelter Missbildung ist sehr selten und in der Litteratur finden wir nur wenige gleiche Beispiele.

Camille Dareste (1, pag. 528, Fig. 7) hatte zu seiner Verfügung nur ein einziges solches Exemplar, welches jedoch weniger entwickelt war und sich schon im Zustande der Zerlegung befand.

Der dritte Fall von Gerlach (2, pag. 32, Taf. III Fig. 2) bietet anch eine bedeutende Ähnlichkeit mit dem unsrigen, ist aber etwas älter.

Dasselbe kann man auch von der Doppelbildung am Staarenei sagen, welche KLaussner beschrieben hat ( 3 , pag. 48, Taf. $X$ Fig. 56-58).

Endlich hat in der letzten Zeit Erich Hofrmans den Fall eines seltenen Anadidymus des Hühnchens veröffentlicht, welches jünger als das unsrige ist, aber demselben nebst den vorhergehenden Beispielen am meisten gleieht (7, pag. 40-61, Taf. VIII).

\section{Das Nervensystem.}

Die Anlage des centralen Nervensystems im Kopf des ersten Embryos $(A)$ stellt von der Oberfläche eine deutliche Zergliederung in drei Theile dar: das primäre, stark entwickelte Vorderhirn-, das Mittelhirn- und das Hinterhirnbläschen. Die Ränder des Nervenrohres kommen, vom Gebiet des Mittelhirns an bis zum Anfang des Hinterhirns, wie man es auf den Querschnitten 1) sieht (Taf. XIV Fig. 12 u. 13), zusammen, fließen aber nicht ganz zusammen; von hier nach hinten gehen sie allmählich bis zum Schwanzende des Embryos aus einander (Taf. XIV Fig. 14 u. 16).

Im Anfangs weit offenen Nervenrohr des zweiten Embryos, dann im Gebiet, welches dem zukünftigen Mittelhirn entspricht, nähern

1) Das Blastoderm des Embryos war in $3 \% \mathrm{HNO}_{3}$ abgenommen und mit KLeinenberg's Hämatoxylin gefärbt. Die Sehnitte sind vermittels MrNot's Mikrotom gemacht und haben eine Dicke von $1 / 300 \mathrm{~mm}$. Die Fläche der Schnitte ist zur langen Achse der hellen Keimflüche perpendikulär und folglich zar Achse des größeren Embryos nicht streng perpendikulär, da letztere davon etwas auf die Seite abweicht. 
sich die Ränder einander, fließen aber nirgends vollständig zusammen, so dass die Nervenplatte hier tuberall wie eine wirkliche Rinne aussieht.

In Folge der schwachen Krümmung nach rechts des ganzen Kopftheils des größeren Embryos $(A)$ ist die rechte Wand des Gehirns etwas schwächer entwickelt.

Die Vereinigung der Nervenplatte des kleinen Embryos $(B)$ mit dem Hirnrohr des größeren geschieht im Gebiet des Vordertheils des Hinterhirns des letzteren, so dass die rechte Wand dieses Hirns auf einer bedeutenden Strecke schon als der Rand der gemeinsamen Medullarplatte erscheint, welche auch den ganzen gemeinsamen Theil der Achse der beiden Embryonen auf der ganzen Strecke bis zum Reste des Primitivstreifens zudeckt.

Die Ränder dieser Medullarplatte sind etwas gehoben und übertreffen bedentend an Dicke das nächste Ektoderm. Auch der Mitteltheil der Medullarplatte (Textfig. 5), welcher der Scheidelinie zwischen den Zwillingen entspricht, ist gehoben. Über der Chorda beider, bis zum Schwanztheil, vertieft sich die Nervenfurche.

Bemerkenswerthe Eigenthümlichkeiten bieten die Kopftheile des centralen Nervensystems, besonders bei dem größeren Embryo.

Erstens beobachtet man vor dem Mittelhirn einen asymmetrischen Vorsprung des Hirnbodens nach unten (Textfig. 2). Dieser Vorsprung ist der Reihe nach auf sechs Schnitten sichtbar und schließt sich von der einen Seite unmittelbar an die obere Wand des Kopfdarmes an, und von der anderen an die äußere Wand der mittleren Vertiefung, welche durch die Wände des Infundibn-

Fig. 2.

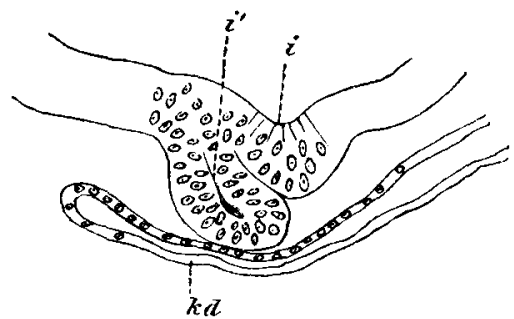

Querechnitt durch den Boden des Zwischenhirns des größeren Embryos $A$ (Taf. XIV Fig. 9, 2). $i$ Infundibulum ; $i$ seine accessorische Aussackung; $k d$ Kopfdarm. lums gebildet wird; man kann in diesem Vorsprung einen spaltenförmigen Raum sehen, und seine änßere Wand ist etwas dicker als diejenige, welche sich an die Wand der erwähnten Vertiefung schließt. Auf dem Schnitte, welcher dem auf der Textfigur 2 vorhergeht, sieht man die unmittelbare Verbindung dieses Vorsprungs mit dem Trichter (Infundibulum) des Zwischenhirns.

Augenscheinlich hatten wir in diesem Fall mit einer besonderen 
und asymmetrischen Entwickelung dieses letzteren nach rechts zu thun, was eher auf die Beziehungen deutet, welche man bei der Bildung des Infundibulum bei den niedrigeren Wirbelthieren beobachtet.

Von der Bildung der Hypophysis cerebri kann in diesem Fall noch nicht die Rede sein in Folge des Alters unseres Embryos und der Differenzirung in diesem Gebiet des Entoderms.

Eine andere Eigenthümlichkeit besteht in der Bildung einer klar ausgedruckten Falte im Gebiet des Mittelhirns, auf der Grenze zwischen der Wand des Hirns und dem Ektoderm. Diese Falte (Taf. XIV Fig. $11 \mathrm{~N}^{\prime}$ ) stammt augenscheinlich von derselben Wand des Hirns und hat eine direkte Beziehung zu der Bildung der ersten Anlage der Gruppe des Trigeminus.

Auf demselben Schnitt bei dem kleineren von den Embryonen (Taf. XIV Fig. 10) bietet die Hirnplatte nur eine Rinne in der Mitte, während ihre Ränder horizontal abgebogen sind, so dass das Ektoderm darunter eine Falte bildet (Taf. XIV Fig. $10 e c$ ). In diesen Rändern eben bemerkt man die Differenzirung, welche zur Bildung der Anlage des peripherischen Nervensystems führt $\left(n^{\prime}\right)$. Wie man es auf dem linken Rande der Zeichnung sieht, gehört diese Anlage doch gänzlich der Hirnplatte an und ist klar vom Ektoderm abgegrenzt, obgleich es hier keine Falte bietet.

Man beobachtet die Anlage des peripherischen Nervensystems auf bedeutender Strecke, aber weiter caudalwärts hat sie nicht mehr den Charakter der oben beschriebenen Falte.

Auf der Fig. 12 (Taf. XIV) ist der rechte (in Wirklichkeit der linke) obere Rand des geschlossenen Nervenrohrs des größeren Embryos $(A)$ ununterbrochen mit dem oberen linken (in Wirklichkeit rechten) Rande der Nervenrinne des kleineren $(B)$ vermittels des ihre Fortsetzung bildenden Ektoderms (e) verbunden. Letzteres bildet fast auf der Mitte dieser Verbindungsbrücke eine ritzenartige Vertiefung (Fig. 12*), welche die Gebiete der Zwillinge nicht ganz abgrenzt. Auf den Schnitten des geschlossenen Nervenrohrs des ersten Embryos befindet sich zwischen dem sich daran anschließenden Ektoderm und seiner äußeren Rückseitenfläche eine fast dreieckige Zellengruppe, welche aus lockeren Elementen besteht, die die Anlage des peripherischen Nervensystems vorstellen (Fig. $12 n^{\prime}$ ).

Der auf der Fig. 13, Taf. XIV, dargestellte Sehnitt geht durch den hintersten Theil des Mittelhirns des größeren Embryos $(\boldsymbol{A})$. Hier verändert sich das Bild in der Hinsicht, dass das Hirnrohr des ersten Embryos sich zu öffnen beginnt; die verdickten Ränder 
des Centralnervensystems beider Embryonen fangen an, sich in der horizontalen Richtung zu einander auszustrecken; und in Folge dessen scheint der sich hier gebildete Keim des peripherischen Nervensystems an die oben genannten ausgezogenen Ränder aufgehängt zu sein (Fig. $13 n^{\prime}$ ).

Die Zellen des ganzen Bezirks, welcher die Keime des peripherischen und die Ränder des Centralnervensystems einschließt, sind sehr locker, so dass die Grenzen zwischen den beiden Bildungen und auch zwischen der Anlage des peripherischen Nervensystems und dem anliegenden Ektoderm, welche auf den vorhergehenden Schnitten klar sichtbar waren, hier undeutlicher werden.

Übrigens kann hier außerdem keine scharfe Grenze zwischen den Keimen des peripherischen Nervensystems beider Embryonen und dem von ihnen eingeschlossenen Ektoderm sein, weil das letztere nach vier Schnitten von dem auf der Fig. 13 vorgestellten ganz verschwindet und die Nervenrinuen der beiden Embryonen mit seinen anrührenden Rändern zusammenfließen.

Die Fig. 14, Taf. XIV, repräsentirt einen der ersten Schnitte, welche auf den zusammengewachsenen Zustand der beiden Nervenrinnen deuten; dieser Schnitt ist durch den Anfang des Hinterhirns des größeren Embryos gegangen. Man sieht darauf, dass das Ektoderm eigentlich zwischen beiden Embryonen gar nicht vorhanden ist und die benachbarten Wände der geöffneten Nervenröhre (Medullarrinnen) gewölbartig eine in die andere gehen.

Die Dicke dieses Gewölbes ist anderthalbmal größer als die der Nervenrinnen; das hängt hauptsächlich davon ab, dass der untere Theil des Gewölbes auf bedeutender Strecke den Keim des peripherischen Nervensystems bildet, welches hier, oder vielleicht etwas mehr nach vorn, seine größte Entwickelung erhält. In demselben Zustande befinden sich einige vorhergehende und weitere Schnitte, wie man aus der Figur 15, Taf. XIV, sehen kann.

Weiter caudalwärts werden die Dicke und die Breite des Gewölbes geringer, aber die Unebenheit seiner unteren Oberfläche und die große Lockerheit der sich darauf befindenden Elemente deuten unzweifelhaft auf das Vorhandensein auch hier des aus den lockeren Zellen gebildeten Keimes des peripherischen Nervensystems (Taf. XIV Fig. $16 n^{\prime}$ ); besonders klar sieht man es bei starker mikroskopischer Vergrößerung.

Noch weiter zurïck wird dieses Gewölbe enger, die Lockerheit seiner Elemente verliert sich allmählich, die untere Oberfläche wird 
schärfer begrenzt und nach $10-15$ Schnitten erscheint die ganze Masse der Medullarplatte mehr oder weniger einförmig.

Wo muss man denn am Anfang des Auftrittes des gemeinsamen Keimes des peripherischen Nervensystems beim Hühnchen suchen, besser gesagt, ist der Rand des Centralnervensystems die Stelle seiner ersten Anlage, oder ist dieser Keim ein Derivat von dem undifferenzirten Theil des ektodermischen Streifens, welcher an das Centralnervensystem grenzt?

Soeben haben wir gezeigt, dass in unserem Fall im Gebiet des Mittel- und Hinterhirns des größeren Embryos, etwas über der Stelle der Vereinigung der Hirntheile der beiden Embryonen, die letzten Spuren der zwischen ihnen eingeschlossenen ektodermischen Platte verschwunden sind, und unterdessen bleibt auf den Schnitten noch auf einer beträchtlichen Strecke unten (20-25 Schnitte) der Keim des peripherischen Nervensystems in demselben früheren Zustande, wie man es sehen konnte, als die Hirntheile der Embryonen durch die oben genannte ektodermische Platte gesondert waren. Also ist der Einfluss des einen von den vermutheten Erzeugern hier zufällig und ganz entfernt, und das hat jedoch das frühere Bild nicht verändert: der Keim des peripherischen Nervensystems verschwindet nicht mit dem Verschwinden des Ektoderms und verändert sich sogar nicht, und daraus folgt, dass die wahren Bedingungen seiner Bildung in demselben Zustande geblieben sind wie bevor und sich auch nicht verändert haben; mit anderen Worten, dass nur die Elemente des Centralnervensystems als einzige und wirkliche Quelle des Keimes des peripherischen Nervensystems beim Hühnchen betrachtet werden mïssen.

Die óben angeführte Falte (Taf. XIV Fig. $11 N^{\prime}$ ) in dem Gebiet des Mittelhirns ist der Vordertheil der Anlage der Trigeminusgruppe und dentet auch direkt auf deren unmittelbare Abkunft von den Wänden des Hirns. Die Art der Bildung selbst des Keimes des peripherischen Nervensystems in Form einer Falte muss als ein Fall der wahrscheinlichen Rückkehr zu den primären Verhältnissen betrachtet werden. Von der Wahrscheinlichkeit dieser Voraussetzung iiberzengt auch HoFFMars's Forschung (8); seinen Artikel habe ich zu dẹ Zeit studirt, als die entsprechende Zeichnung: (Taf. XIV Fig. 11) fertig war.

Hohwans meint, dass, seinen Beobachtungen über die Entwickelung des Haifisches (Acanthias) gemäß, die segmentalen Kopfnerven 
und die hinteren Wurzeln der Rückennerven wie paare segmentale Aussttilpungen des Hirnrohrs selbst gebildet werden und desshalb in ihrer Entwickelung im Allgemeinen den Augenblasen ähnlich sind. Diese Beobachtung ist von großem theoretischen Werth, da sie auf den gemeinsamen Weg der Phylogenese für alle Kopfnerven deutet. Acanthias hat augenscheinlich die primären Beziehungen behalten, welche bei den zu dieser Gruppe nächsten Vertretern sich schon verändert haben, wie ich mich persönlich aus dem Studium der Embryonen von Raja und Pristiurus uiberzeugt habe; die Acanthias-Embryonen, welche sich zu meiner Verfügung befanden, waren schon zu entwickelt ( $7 \mathrm{~mm}$ lang und mehr), um die erste Anlage des peripherischen Nervensystems daran studiren zu können.

Eine andere theoretische Bedeutung der erwähnten Beobachtung Hoffmann's ist die, dass er bestimmt, wo man die Quelle der Bildung des peripherisehen Nervensystems suchen muss, und, in Übereinstimmung mit den von mir seiner Zeit entwickelten Erwägungen (9, pag. 42) das Bild der Entwickelung des Keimes des peripherischen Nervensystems bei Sauropsida für sekundär hält. Die von mir bei der doppelten Missbildung des Hühnchens, welche wir studiren, angezeigte Falte im Gebiet des Keimes der Trigeminusgruppe ist augenscheinlich eine atavistische Erscheinung - die Rückkehr zum primitiven Typus.

Die Chorda dorsalis und das Mesoderm.

Bei dem größeren Embryo bietet die Chorda dorsalis in ihrer Bildung keine sichtbaren Abweichungen. Sie dehnt sich seiner ganzen Länge entlang, und nur an ihrem vordersten Ende beobachtet man eine Zerspaltung, wie man es auf der Textfigur 3 (ch) sieht. Dieses vordere Ende der Chorda ist zum ersten Male nach einem Schnitte candalwärts, nach dem oben beschriebenen Vorsprung des Infundibulum sichtbar. Die Zeichnung 3 ist fünf Schnitte zuriick nach der Zeichnung 2 abgenommen. In der Wand des Infundibulum (Textfigur $3 i$ ), welche sich über dem vorderen Ende der Chorda befindet, bemerkt man zahlreiche Figuren derkaryokinetischen
Fig. 3 .

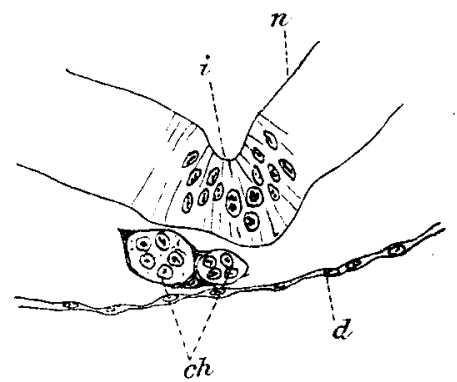

Fūnf Schnitte rūckwārts nach dè Text. figur 2. Ejn Theil des Infundibulum des Embryos $A$ (Taf. XIV Fig. 9, 3). i Infundibulum; $n$ Nervenrohr ; $d$ Darmwand; ch Chordaanlagen. 
Theilung. Die Zerspaltung der Chorda wird nur anf einem Schnitte beobachtet; auf den folgenden erscheint sie schon ganz und grenzt eng an das Entoderm der oberen Wand des Kopfdarmes $(d)$, welehe in diesem Gebiet sehr dünn ist.

Die Chorda des kleineren Embryos $(B)$ hat im Allgemeinen keine bedeutende Länge und muss besonders beachtet werden.

Ihre erste Anlage ist ungefähr auf der Strecke eines Drittels der Länge des Körpers vom Kopfe sichtbar, während vorn an ihrer Stelle einzelne Zellen des embryonalen Bindegewebes oder Somiten sich befinden. Es ist interessant, dass die Rudimente der Chorda hier eng mit dem Entoderm verbunden sind (Textfigur 4).

So sehen wir in dem ganzen vorderen Theil, dass sich rom Entoderm einige Zellen abtheilen, welche sich Anfangs augenschein-

Fig. 4.

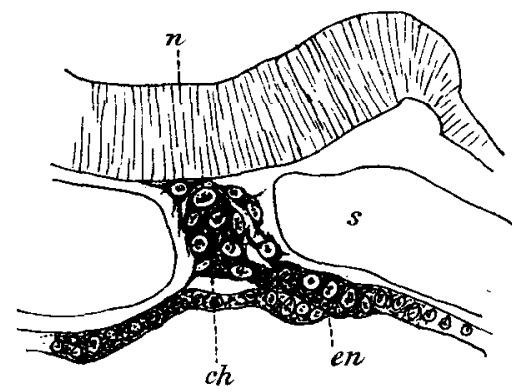

Die vordere Anlage der Chorda des kleineren Embryos $B$ (Taf. XIV Fig. 9, 4). $n$ Medullarplatte; $s$ Somit; ch Chorda dorsalis in Verbindangimit dem Entoderm $\epsilon n$. lich in Form einer Falte oder einer rinnenartigen Vertiefung abgesondert haben; darauf deutet rechts eine doppelte Reihe Zellen im Entoderm, eben an der Stelle, wo die primitive Verbindung mit der Chorda sich noch erhalten hat (Fig. 4 en). Die Chorda selbst ist nicht eine Bildung, welche von der Peripherie begrenzt wäre, und ist noch in zwei Stellen mit dem Entoderm verbunden.

Der hier beschriebene Keim der Chorda ist nur noch auf vier Schnitten sichtbar; nach vier Schnitten ohne Chordaanlage in der Richtung des Schwanzendes erscheint wieder die Chorda; hier hat sie eine typischere Struktur und ist auf sieben Schnitten sichtbar. Weiter nach hinten verschwindet sie wieder und wird durch unpaare Somiten ersetzt.

Der zweite Theil der Chorda erscheint als echte Falte des Entoderms (Textfiguren 5 u. 6), von welchem sie sich sogar nicht abtheilt, und ist eigentlich eine typische Chordarinne (Textfigur $6 c h^{\prime}$ ), wie es z. B. Hoffmans bei Haematopus ostralegus, Sterna paradisea ete. dargestellt hat $(\mathbf{1 0})$. So haben wir hier wieder mit einer unregelmäßigen Abweichung in der Richtung der primären Verhältnisse zu thun.

Am hinteren Ende des Körpers der beschriebenen Doppelbildung, 
bei dem kleineren Embryo, anstatt der Chorda, hinter den Somiten, beobachtet man nur einzelne mesodermische Zellen.

Beide Embryonen haben je sechs Paar Somiten, doch sind dieselben bei dem größeren klarer ausgedrickt, und bei dem kleineren sind die letzten drei Paare von drei unpaaren Bildungen vorgestellt (Textfig. 5); die Individualität der einzelnen Somiten kann doch in diesen Bildungen gewissermaßen ausgesprochen sein (Textfig. $7 s$ ).

In der mesodermalen Masse, welche sich rom Fig. 5.

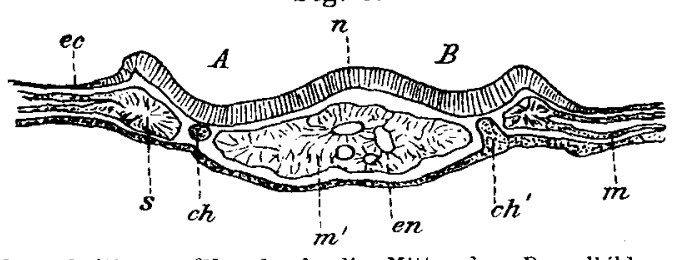
Querschnitt nngefähr durch die Mitte der Doppelbildung (Taf. XIV Fig. 9, 5). ch Chorda des größeren Embryos(A); $c h^{\prime}$ die des kleineren $(B)$. ec Ektoderm, en Entoderm, $m$ Mesoderm. Die Medullarplatte $(n)$ ist in der Mitte anfgehoben und deckt die zusammengeflossene mesodermale $\mathrm{Mass}\left(m^{\prime}\right)$ mit einigen Zusammenfließen der beHöhlen ; s Somit. nachbarten Somiten und der Splanchno- und Somatopleura gebildet hat, beobachtet man Räume, welche unregelmäßig gelegen und an Zahl versehieden sind (Textfigur $\left.5 m^{\prime}\right)$. Auf der Fig. 9, Taf. XIV, welche die Doppelbildung von der Rückenseite in toto darstellt, kann man die genannte Masse in Form eines dunklen Streifens beobachten, welcher mit seinem breiten Ende unweit vor den Somiten beginnt und sich bis zum hinteren Ende der Medianlinie der Doppelbildung entlang Fig. 6.

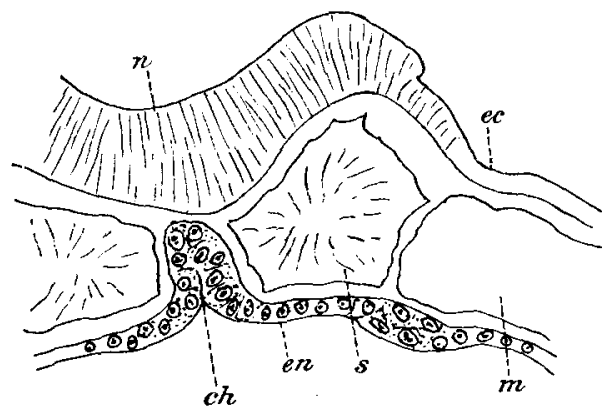

Von demselben Selnitt, wie Textfig. 5, bei bedeutenderer Vergrößerung. Die Chordaanlage des kleineren Embryos $(\boldsymbol{B})$. Die Bezeichnungen wie anf der Textfigar 5 . zieht.

\section{Das hintere Ende des Zwillingspaares.}

Dieser Theil ist in der Beziehung interessant, dass hier eigentlich die genetische Abhängigkeit der beiden Embryonen von einander bestimmt wird. Wie es oben gesagt worden ist, erreicht bei dem kleineren Embryo die Chorda nicht das hintere Ende des Körpers; ebenfalls sind die ihm gehörenden Theile der Medullarplatte 
fast nicht abgesondert (Textfig. 7), während bei dem größeren Embryo man die der Länge nach laufende Vertiefung - - die Rtickenfurche - über der Chorda eben so lange, wie die letztere, beobachtet.

Fig. 7.

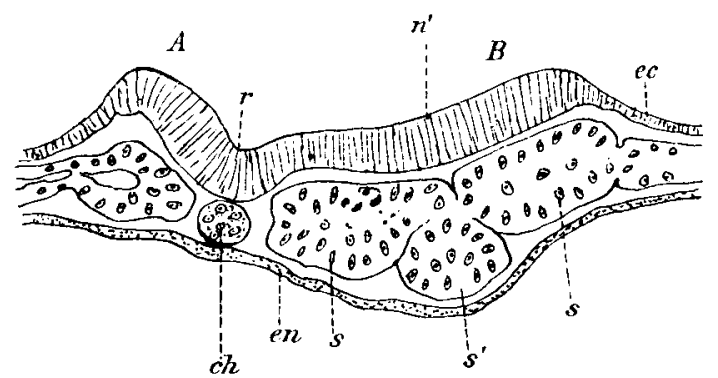

Querschnitt durch das hintere Drittel der Doppelbildung (Taf. XIV Fig. 9, ). $n$ asymmetrische Medullarplatte, welehe für die beiden Embryonen $A$ und $B$ gemeinsam ist; ch Chorda; $s$, $s^{\prime}$ Somiten; r Rũckenfurche.

In Folge dessen erscheint die Medullarplatte auf dem Querschnitt asymmetrisch (Textfig. $7 n^{\prime}$ ); wenn wir uns aber dem hinteren Theil der Chorda des größeren Embryos nähern, wechselt das Bild und die Medullarplatte wird immer mehr symmetrisch.

Fig. 8.

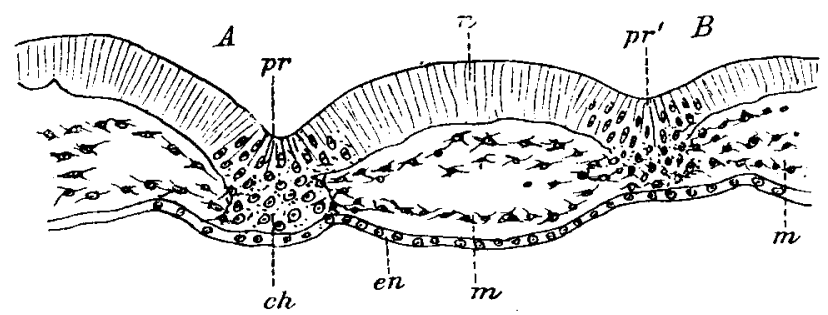

Querschnitt durch den hinteren Theil der Doppelbildung (Taf. XIV Fig. 9, 8). $u$ Medullarplatte; ch Chorda; $m$ Mesoderm; en Entoderm; pr Primitivrinne des größeren Embryos $A ; p r$ dieselbe des kleineren $(B)$.

Die erwähnte Rückenfurche des größeren Embryos wird kleiner und sodann durch eine neue, tiefere ersetzt, neben welcher bald auch eine andere erscheint. Diese beiden auf den Schnitten neuerschienenen Rinnen (Textfig. 8) gehören unbestreitbar den Primitivstreifen an. Die erste gehört dem größeren Embryo $(A)$, die zweite dem kleineren $(B)$. Die Textfig. 8 stellt einen Schnitt vor, weleher ungefähr durch das untere Drittel des Primitivstreifens des 
Embryos $A$ und durch das obere Drittel des Primitivstreifens des Embryos $B$ geht. Beide Rinnen haben fast die gleiche Länge, nur ist die erstere etwas tiefer und die zweite schwächer ansgedrückt. Beide stehen in einem spitzen Winkel zu einander. In Folge ihrer verhältnismäßig schwachen Differenzirung waren sie auf der Doppelbildung in toto nicht genügend bestimmt worden. Die Chorda des größeren Embryos (Textfig. $8 c h$ ) verliert allmählich an ihrem hinteren Ende ihre bestimmten Umrisse und fließt mit der gemeinsamen Zellenmasse zusammen, welche in Vereinigung mit dem Ektoderm ist und den Boden der Primitivrinne darstellt. Das Entoderm erscheint auf diesem Schnitte von der erwähnten Zellenmasse ganz abgesondert und nur die Grenzlinie zwischen ihnen ist sehr klar sichtbar. Dasselbe kann man auch bezüglich der Primitivrinne des kleineren Embryos sagen, dessen Proliferation der Zellen vom Boden überhaupt schwächer ausgedrückt ist (Texfig. $8 \mathrm{pr}^{\prime}$ ). Die entsprechende Zellenmasse, von welcher gewöhnlich die Chorda nach vorn wächst (im gegenwärtigen Fall ist die letztere in diesem Theil gar nicht ausgedriickt, hat bier doch mehr oder weniger bestimmte Umrisse und schließt sich eng an das Entoderm auf bedeutender Strecke an, ohne jedoch mit ihm zusammenzufließen.

Fig. 9.

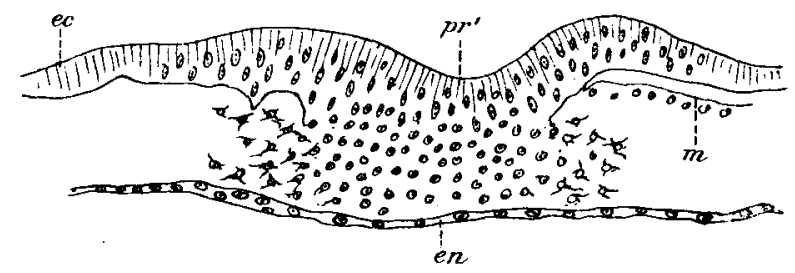

Querschnitt durch den hintersten Theil der Doppelbildung (Taf. XIV Fig. 9, 9). Der Primitivstreifen ist nur von dem kleineren Embryo geblieben $\left(p r^{\prime}\right)$. Dje Bezeichnungen wie auf der Fig. 8.

Etwas weiter zurtick kommen beide Primitivstreifen zusammen und scheinen in einen zusammenzufließen, wobei die Rinne des größeren Embryos, welche hier endigt, eine unterworfene Rolle bezïglich derjenigen des kleineren spielt, welche letztere sich nach hinten noch auf einer bedeutenden Strecke fortsetzt. Auf der Textfigur 9 sehen wir sie noch klar ansgedrïckt $\left(p r^{\prime}\right)$, während vom Primitivstreifen des größeren Embryos keine Spur geblieben ist.

Dieser Umstand zeigt deutlich, dass das Zwillingspaar, welches wir beschreiben, aus zwei ganz selbständigen Keimen entstanden ist; welche in Form von Primitivstreifen noch selbstïndig waren, 
wobei der Primitivstreifen des kleineren etwas hinter dem des größeren lag, und letzterer seinerseits zu dem ersteren in einem Winkel gelegen war. In der Folge vereinigte sich das hintere Ende dieser Furche mit derjenigen des kleineren Embryos und war in seiner folgenden Entwickelung selbständig, in wie fern es die engen topographischen Verhältnisse erlaubt haben.

\section{Von den Ursachen der Bildung von Doppel- und Melrfachbildungen.}

Wie mannigfaltig auch auf den ersten Blick die vier beschriebenen Fälle der Missbildungen des Hühnchens in der ersten Entwickelung sind, haben sie doch viel Gemeinsames, oder, besser gesagt, es besteht dazwischen eine direkte genetische Abhängigkeit, wodurch die Gruppirung des angeführten Materials erklärt wird.

Der erste (Textfig. 1; Taf. XIII Fig. 1) und der dritte (Taf. XIV Fig. 7) Fall (wenn wir in dem letzteren die Nebenkomplikationen nicht beachten) gleichen sich in der Hinsicht, dass beide doppelte Primitivstreifen haben, welche in einem Fall vermittels ihrer vorderen Enden sich einander nähern und im anderen durch ihre hinteren Enden zusammenkommen. Als direktes Resultat der folgenden Entwickelung des ersten Falles erscheint der zweite Fall (Taf. XIII Fig. 2), als sichtbar mögliche Folge des dritten erscheint der vierte (Taf. XIV Fig. 9).

Die Vergleichung dieser Fälle zeigt uns, wo wir die Gründe der Bildung doppelter Missbildungen suchen müssen. Es ist klar, dass diese Ursachen sich in den ersten Phasen der Entwickelung finden und von einem primären Doppeltsein des Keimes selbst und nicht von der späteren Zerspaltung des Anfangs einfachen Keimes abhängen. Was aber ruft das Doppeltsein oder sogar die größere Anzahl der Keime in den allerersten Entwickelungsphasen hervor? Hängt das Erscheinen dieser Anomalien von den Eigenschaften des unbefruchteten Eies selbst ab, von welchem sie stammen, oder von Veränderungen in dem noch nicht differenzirten Keime?

Die Lösung dieser Alternative nur im Sinne des ersten Falles entspricht einer Ausweichung der Lösung der Aufgabe. Andererseits aber, sind denn irgend welche Gründe, welche die Möglichkeit geben, eine solche äußerste Veränderlichkeit im noch nicht differenzirten Keime zu vermuthen? Es giebt solche Gründe. Vor 
Allem findet die Thatsache des Vorhandenseins des Primitivstreifens in doppelter Zahl und mehr einen gewissen Wiederhall in den $\mathbf{A b}$ weichungen der Primitivstreifen, wenn sie einzeln erscheinen.

\section{Abweichungen in der Bildung einzelner Primitivstreifen.}

In sehr vielen embryologischen Arbeiten deutet man darauf, dass besonders beim hinteren Ende des Primitivstreifens beim Hühnchen die verschiedensten Veränderungen vorkommen. Sogar in M. Duval's Atlas $(\mathbf{1 1}$, pl. IV, V), welcher am wenigsten monströse Abweichungen in Augenschein hat, haben wir Darstellungen, auf denen der Primitivstreifen mit Krümmungen, Seitenvorsprïngen und sogar Zerspaltungen erscheint (Fig. 65, 66, 68, 76, 84). Viel mehr solcher Abweichungen finden wir bei KUPFFER und BeNECKE (12, Taf. II-VIII Fig. 9, 18, 26, 39, 43, 47) auf Keimscheiben des Hühnchens und des Sperlings.

Das Material, welches in dem Augenblicke zu meiner Verfügung war 1), hat auch zahlreiche Abweichungen in der Bildung des Primitivstreifens dargeboten, Abweichungen, welche in direkte oder indirekte Verbindung mit den oben beschriebenen Fällen der Missbildungen gestellt werden können.

Als Anfang solcher Abweichungen kann ein Primitivstreifen betrachtet werden, dessen Ränder auf derselben Strecke uneben sind und eine Art Seitenausschnitte bilden, wie man es auf der Fig. 17, Taf. XIV, sieht.

In diesem Fall sind diese Ausschnitte und Unebenheiten der Umrisse des hinteren Theils der Primitivrinne sehr klein, was die Länge betrifft, aber sehr tief und scharf ausgedrückt. Gewöhnlich entwickelt sich die Keimscheibe mit einem solchen Primitivstreifen normal, da man solche Unebenheiten auch auf späteren Entwickelungsstufen sieht, wenn das Nervensystem sich schon bildet und man den Primitivstreifen als rudimentären Rest beobachtet (Taf. XV Fig. 20).

Die gezeigten Abweichungen erklären aber die Herkunft schärfer ausgedrickter Anomalien, wie auch den Fall, welcher auf der Fig. 18, Taf. XV, dargestellt ist, wo das hintere Ende des Primitivstreifens

1) Die Präparate aus dem zootomischen Laboratorium der Universität Warschau, welche persönlich von mir und von Anderen vorbereitet sind, die sich in den letzten fün Jahren mit der Entwickelung des Hïhnchens beschäftigten. 
erweitert und verdickt ist. In dieser Verdickung zerspaltet sich etwas am Ende die Primitivrinne, welche sich kaum bemerkbar fortsetzt und noch früher sich mit zwei auch schwach ausgedrückten Seitenfurchen vereinigt.

Der dritte Fall (Fig. 19, Taf. XV), welchen ich für passend halte jetzt zu zeigen, bietet einen Primitivstreifen, dessen Primitivrinne im hinteren Theil etwas erweitert und auf eine Seite gekrümmt ist $(a b c)$, an der Stelle der Krümmung $(b)$ ist ein kleiner Seitenvorsprung, welcher als der Anfang der Verzweigungen der Rinne an dieser Stelle angesehen werden kann. Dieselbe Verzweigung findet auch am Ende der Primitirinne statt.

Die manchmal bedeutende Erweiterung der Primitivrinne kann auch in der späteren Entwickelung bestehen, wie man es auf der Fig. 20, Taf. XV, sieht, wo bei einem Embryo ein anormaler Primitivstreifen mit scharf ausgedrückten Medullarwülsten und der

Fig. 10.

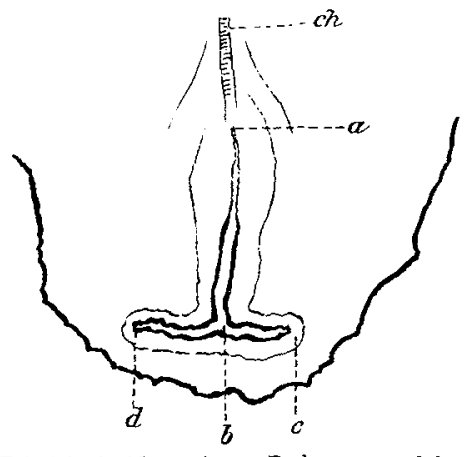

Primitivstreifeu eines Embryos, welcher fün abgesonderte Somiten hatte und im Algemeinen anormal war. Das Kopfende ist abgesondert; die Medullarplatte ist auf der ganzen Ausdehnung breit und seine Rānder sind auf dem Wege zum Zusammenflieben nur aufgehoben. Dis C'horda fängt vom Primitivstreifen an, wird in der Mitte enger und in Vordertheile breiter. Ihr vorderes Ende dehnt sich bis zur Medianausbuchtang des vorderen Randes der Medullarplatıe. Die Lānge der Medullarplatte beträgt vom vorderen Rande bis zum ersten Somit $1,6 \mathrm{~mm}$, die des Primitivstreifens bis zur Verzweigung ( $a b) 1 \mathrm{~mm}$; die der Zweige $b c 0,25 \mathrm{~mm}$, der Zweige $b d$ $0,25 \mathrm{~mm}$. ch Chorda.

Eine solche Anomalie kann man als eine unregelmäßige Entwickelung des hinteren Endes betrachten, andererseits aber scheint
Kopffalte dargestellt ist. Das hinterste, sich nach links richtende Ende dex Primitivrinne ist schwächer ausgedruickt und besitzt zahlreiche Seitenvorspriunge.

Die oben angedeutete Neigung des Primitivstreifens, Seitenvorsprünge zu bilden, kann in einem hohen Grade ausgedriuckt sein und zum Erscheinen seiner wirklichen Verzweigungen führen.

In dem auf der Fig. 21, Taf.XV, dargestellten Fall sehen wir die klare Zerspaltung des hinteren Endes des Primitivstreifens, welcher hier wie ein Anker aussieht; leider war auf dem Präparate diese Theilung theilweise rom Dotter zugedeckt.

Dieselbe Eigenthümlichkeit ist viel mebr auf der Textfigur 10 (Photogramme Taf. XIII Fig. 4) ausgedrückt, welche von einer Keimscheibe späterer Entwickelung abgebildet ist. 
sie drei Primitivstreifen darzustellen, welche in einem Punkte aus einander gehen, wobei nur der mittlere sich normal entwickelt hat. Im gegenwärtigen Fall geschieht das nicht und die Horizontalrinne (dc) kann eher als atavistische Erscheinung betrachtet werden, welche hier auf eine zufällige Entwickelung des Urmundes (des Sichels) in der Form, wie derselbe den Reptilien eigen ist, deutet.

Es ist aber kaum möglich ebenso den auf der Textfigur 11 Photogramme Taf. XIII Fig. 5) dargestellten Fall zu erklären. Hier ist das hintere Ende des Primitivstreifens auch zerspaltet, diese Zerspaltung kann aber eher, wie darauf die Umrisse der Area pellucida deuten, als das Resultat des Znsammenfließens zweier Primitivstreifen betrachtet werden, von denen der in einem stumpfen Winkel zu dem rechten gelegene linke $(b d)$ in seiner Entwickelung stehen geblieben ist, und der rechte $(a b c)$ den Anfang der Embryo-Entwickelung gegeben hat.

Auch kann augenscheinlich das Wachsthum der Primitivstreifenanlage in den verschiedensten Richtungen und seine vorwiegende Entwickelung in der einen stattfinden; in Folge dessen können die Verzweigungen am hinteren Ende bedeutender sein. Auf der Fig. 22, Taf.XV, sind solche Verzweigungen kaum dargestellt und

Fig. 11.

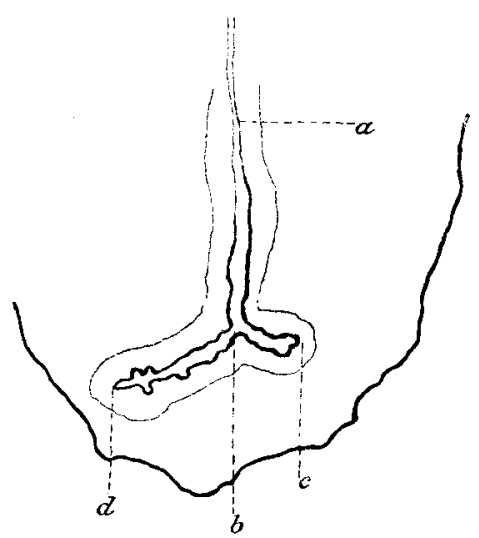

Der Primitipstreifen des normal entwickelten Embryos mit 5 gesonderten Somiten. Die Medullarwâlste nähern sich einander in der Gegend des Mittelhirns. Die Länge der Medullarplatte bis zum ersten Somit betrăgt $1,85 \mathrm{~mm}$; die des Primitivstreifens bis zur Verzweigung $(a b) \quad 1,16 \mathrm{~mm}$; die der kleineren $\mathrm{Z}$ weige $(b c) 0,20 \mathrm{~mm}$; die der größeren (bd) $0,60 \mathrm{~mm}$. schließen sich direkt an den ersten und den zweiten (Taf. XIV Fig. 17, Taf. XV Fig. 18) von den Fällen, welche oben beschrieben sind.

Sie können aber auch sehr bedeutend ausgedrückt sein, so dass jedes Zweiglein den Charakter einer wirklichen Primitivstreifenanlage haben kann. Auf der Textfigur 12 (Photogramme Taf. XIII Fig. 6) haben wir einige solche Verzweigungen, and die eine ( $b d=$ $0,50 \mathrm{~mm}$ ) erreicht eine verhältnismäßig bedeutende Länge.

Es wird hier passend sein, auf die strangförmigen Ausstrahlungen des Primitivstreifens zu deuten, welche Er. Hoffmann beschrieben hat (7, pag. 56, Fig. 1 u. 12). Welches Schicksal eine solche 
Fig. 12.

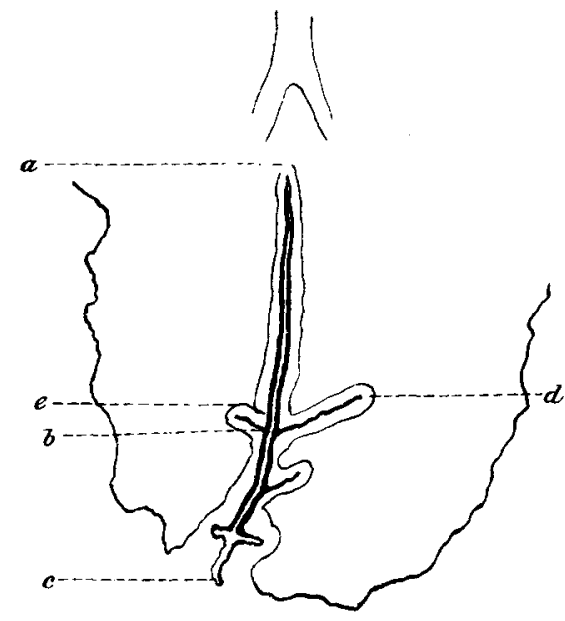

Keimscheibe von 22 Stunden der Bebrütung. Die ganze Länge des Primitivstreifens $(a b c)$ 2,40 $\mathrm{mm}$; die der größeren Zweige $(b d) \quad 0,50 \mathrm{~mm}$; die der kleineren $(b e) \quad 0,25 \mathrm{~mm}$.
Verzweigung oder die Rudimente des Primitivstreifens in der folgenden Entwickelung haben können, das müssen direkte Beobachtungen zeigen, welche wir noch nicht zu unserer Verfügung haben. Doch ihr Vorhandensein auf mehr oder weniger späten Entwickelungsstufen zeigt, dass in dem nicht differenzirten Blastoderm die Zertheilung (oder das Zerfallen auf mehr als zwei Theile des Centrums der primitiven Entwickelung (in Anwendung auf den gegenwärtigen Fall des Primitivstreifens) mit noch größerer Leichtigkeit stattfinden kann.

\section{Der Einfluss der Form der Area pellucida auf die Bildung des Primitivstreifens.}

Es ist unzweifelhaft, dass in der primitiven Entwickelung der Thierformen der Charakter des bildenden Materials eine große Bedeutung hat: es hat einen unmittelbaren Einfluss auf die morphologische Seite der ersten Entwickelungsphasen.

Abgesehen von anderen Bedingungen, haben auf die Form des Primitivstreifens die Umrisse der Area pellucida Einfluss. In den Fällen, wo der Primitivstreifen der Länge nach nicht entwickelt ist, eben so wie im Anfange seiner Bildung, nähern sich die Umrisse der Area pellucida dem Umkreise. In den Fällen jedoch, wo die Area pellucida aus irgend einem Grunde eine unregelmäßige Form bekommt, wird auch der Primitivstreifen missgestaltet.

In gewissem Grade entspricht diese Erscheinung der Auffassung, dass die ersten Entwickelungsprocesse als der Ausdruck für das Zusammenwirken der ganzen Summe der Einflüsse der Bestandtheile des bildenden Materials erscheinen. Wenn sich diese Processe in der Bildung des Primitivstreifens ausdrücken, so muss er 
sich ungefähr in gleicher Abhängigkeit von allen Elementen der Area pellucida, als des nächsten bildenden Materials, befinden; desshalb muss er in der Area pellucida eine mittlere Lage einnehmen, wie es auch in normaler Entwickelung stattfindet.

Wenn aber die Area pellucida die gewöhnlichen regelmäßigen Umrisse nicht besitzt, so muss in entsprechender Weise sich die Form des Primitivstreifens verändern. Der Vorsprung des hellen Fruchthofes von irgend welcher Seite muss entweder einen Theil des Primitivstreifens erhalten, wie es auch in den oben gezeigten Fällen stattfindet (die Abweichung des einzelnen Primitivstreifens, Fig. 20, Taf. XV; Photogramme Taf. XIII Fig. 5), oder einen selbständigen Primitivstreifen, wie es auf den ersten zwei oben beschriebenen Fällen der doppelten Missbildung vorkommt.

\section{Das Erscheinen von zwei oder einigen Primitivstreifen in einer Keimscheibe.}

In dem ersten der von uns beschriebenen Fälle (Photogramme Taf. XIII Fig. 1) sind die Umrisse der Area pellucida ganz anormal, und dem Primitivstreifen wäre es am natürlichsten, diejenige Lage einzunehmen, welche der quasi dritte Primitivstreifen einnimmt, welcher eigentlich der Rest des Keimwalles ist, oder eine perpendikuläre Lage zu demselben. Das geschah jedoch nicht, das Keimfeld ist auf zwei Theile zerfallen und in jedem derselben hat sich ein selbständiger Primitivstreifen gebildet (Textfigur 13), im rechten $(A)$ seiner Länge

Fig. 13.

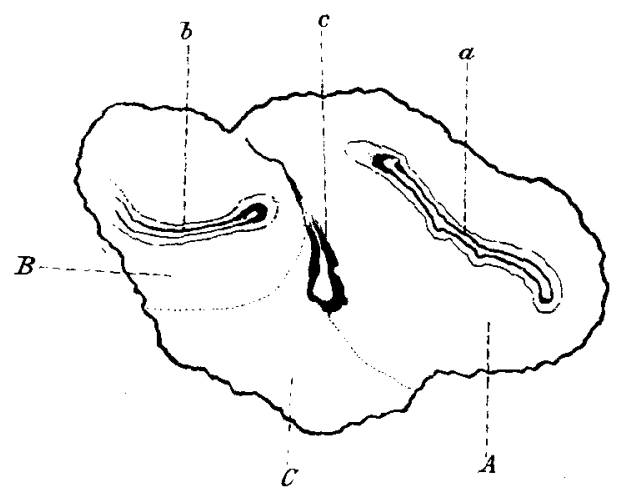

Keimseheite des Hühnchens nach einem Photogramm. $a$ und $b$ Primitivstreifen, $c$ scheinbarer Primitivstreifen von Dotterresten gebildet. $A, B$ Area pellucidae beider Anlagen. $C$ zwischenliegendes Feld ohne Primitivstreifen. nach, im linken $(B)$ ein gekrümmter und nur unter dem Einfluss seiner oberen Hälfte, da die untere, sehr wahrscheinlich, als ein unabhängiger Theil des Ektoderms $(C)$ ohne Furche erscheint. Jedenfalls ist der Winkel, welchen sie einnimmt, bis zu einem gewissen Grade abgesondert, wie man es auch auf der Photographie sieht (Phetogramme Taf. XIII Fig. 1). 
Auf den oben erwähnten Fall von Kuaussner (3, Fig. 60) können dieselben Erwägungen wie zu meinem zweiten (Photogramme Taf. XIII Fig. 2) angewendet werden, welcher schon eine spätere Entwickelungsstufe der hier angedeuteten Beziehungen darstellt.

Wenn wir den Einfluss der Area pellucida auf die Bildung des Primitivstreifens anerkennen, so stellt sich natiirlich die Frage, was seinerseits die Veränderung ihrer Umrisse beeinflusst. Unzweifelhaft hängen die Umrisse des hellen Fruchthofes, erstens von den Richtungen $a b$, in welchen sich die Zellenelemente energischer vermehren, und das hängt seinerseits unmittelbar von den inneren Eigenschaften der primären Bildungselemente $a b$; zweitens von den Bedingungen, welche in anderen Richtungen das Wachsthum zurückhalten.

Leider sind unsere Kenntnisse in diesem Gebiet selnr gering. Es ist unzweifelhaft, dass ein intensiveres und zurückgehaltenes Wachsthum des Blastoderms z. B. vom Zugang des Sauerstoffes abhängt; darauf deuten direkt die Beobachtungen vieler Gelehrten, welche sich mit dieser Frage beschäftigt haben, wie auch meine persönlichen, doch können wir noch nicht genügend den Grad der Abhängigkeit bestimmen. Die unregelmäßige Form des Keimfeldes kann auch das Resultat des Einflusses nicht nur irgend einer Lrsache, sondern deren ganzen Systems sein. Das geschieht fast immer in Wirklichkeit und dadurch wird die Schwierigkeit der Erklärung eines jeden einzelnen Falles verursacht. Wenn aber eine solche Form erscheint, so bietet sie ein Feld für die Entwickelung von Missbildungen mit Doppel- und Mehrfachbildungen, wie es unter Anderem auch in den oben beschriebenen Fällen stattgefunden hat.

Indem ich meine Erwägungen angeführt habe, beabsichtigte ich nicht eine vollständige Hypothese der Herkunft der Mehrfachbildungen zu geben oder die schon in dieser Frage bestehenden Theorien durch andere zu ersetzen. Ersteres fordert ein umfangreicheres Material, das andere eine tiefere litterarische Bearbeitung; doch habe ich die Absicht, beides mit der Zeit darzubieten. Im gegenwärtigen Fall aber möchte ich nur nebst neuen Thatsachen, auf solche Seiten denten, welche bis jetzt in der Lösung der Frage von der Herkunft der Missbildungen nicht genügend beachtet worden sind. 


\section{Litteraturverzeichnis,}

1) Camlle Dareste, Recherches sur la production artificielle des monstruosités. 2ème Édition. 1891.

2) I. Gerlach, Die Entstehungsweise der Doppelmissbildungen bei den höheren Wirbelthieren. 1882.

3) F. Klaussner, Mehrfachbildungen bei Wirbelthieren. 1890.

4) R. Burckhardt, Doppelanlage des Primitivstreifens bei einem Hühnerei. Archiv für Anatomie und Physiologie. Anatom. Abth. 1888.

5) A. Rauber, Die Theorien der excessiven Monstra. Virchow's Archiv, Bd. 71 (1877), 73 und 74 (1878).

6) А. II тицын т, Наблюденія надъ развитіемь периферичсской нерниой системи у цыпленка. Работы изъ Зоотом. Лабор. Варшг. Унив. III. 1892.

7) Erich Hofrmans, Über einen sehr jungen Anadidymus des Hühnchens. Archiv für mikroskop. Anat. Bd. 41, 1893.

8) C. K. Hofrmany, Zur Entwickelungsgeschichte des Selachierkopfes. Anatom. Anzeiger, IX, Nr. 21, 1894.

9) П. Митрофановт, Изслъдовапіх надь развигісмь позвоночныхт жлпотныхт. Раб. Зоот. Лабор. Варш. Ун. I. 1892.

10) C. K. Hoffmans, Die Bildung des Mesoderms, die Anlage der Chorda dorsalis und die Entwickelung des Canalis neurentericus bei Vogelembryonen. (Königl. Akademie der Wissenschaften. Amsterdam.) 1883.

11) M. Duval, Atlas d'embryologie. 1889.

12) C. Kupffer und B. Benecke, Photogramme zur Ontogenie der Vögel. 1879.

\section{Erklärung der Abbildungen ${ }^{1}$ ).}

\section{Tafel XIII. (Photogramme.)}

Fig. 1. Keimscheibe eines Hühnchens mit zwei Primitivstreifen und einem dritten scheinbaren. Näheres in der Erklärung der Textfigur 1.

Fig. 2. Doppelbildung des Hïhnchens mit gemeinsamem Kopfe.

Fig. 3. Der Haupttheil der Keimscheibe mit einigen Primitivanlagen (cf. Taf. XIV Fig. T).

Fig. 4. Der Primitivstreifen eines missgebildeten Embryos des Hühnchens mit fünf Somiten.

1) Die Textfigturen 1, 10-12, Taf. XIV Fig. 7 and 17, Taf. XV Fig. 18-22 sind nach Photogrammen ansgeführt; die Textfiguren 2-9, Taf. XIV Fig. 8, 10, 11 sind von mir mit Camera lncida aufgenommen. Die Figuren 9, 12-16, Taf. XIV, stammen von·A. PTIZIx's Arbeit $(6$, und sind auch mit Camera lucida gezeichnet. 
Fig. 5. Derselbe eines normalen Embryos des Hühnchens mit fünf Somiten, weniger vergrößert.

Fig. 6. Der Primitivstreifen des Hühnchens von 22 Bebrütungsstunden.

\section{Tafel XIV.}

Fig. 7. Keimscheibe des Hïhnchens vom 3. oder 4. Bebriitungstag. $a, b, f$ Primitivstreifen, $c d, e, g$ sekundäre Furchen und Falten, $h$ gemeinsame Verdickung für die Primitivstreifen $a$ und $b ; i$ Ektodermverdickung, $k$ die Grenze des Keimes. $I-V$ zeigen die Lage der Schnitte der folgenden Figur 8. - Nach einem Photogramme. Der centrale Theil mit Primitivstreifen und Furchen ist auf der Fig. 3 der Taf. XIII dargestellt.

Fig. 8. Die Querschnitte durch die Keimscheibe der Figur 7, deren Lage da durch die Ziffern $\mathrm{I}-\mathrm{V}$ gezeigt ist. ec Ektoderm; en Entoderm; w Dotterwall. Die anderen Bezeichnnngen wie auf der Fig. 7.

Fig. 9. Doppelbildung des Hühnchens. Die Ziffern 2-9 zeigen die Lage der Textfiguren 2-9; die Ziffern $X I V, 12 ; X I V, 14 ; X I V, 16-$ die Lage der Figuren 12-16 der Taf. XIV.

Fig. 10 u. 11. Von einem und demselben Schnitte zwei Schnitte rückwärts nach der Textfigur 3). Hirnschnitte des Embryos $A$ (Fig. 11) und des Embryos $B$ (Fig. 10). ec Ektoderm; $n$ Nervenplatte; $n^{\prime}$ Anlage des peripherischen Nervensystems; $N$ Nervenrohr; $N^{\prime}$ Falte zwischen dem Ektoderm und dem Nervenrohr, welche die Anlage der Trigeminusgruppe vorstellt; $c h$ Chorda dorsalis.

Fig. 12. Ein Querschnitt durch die Kopftheile beider Embryonen (Fig. 9). $A$ Mittelhirn des größeren Embryos; $B$ Vorderhirn des kleineren. Zwischen den beiden Embryonen bemerkt man eine Furche. e Ektoderm; en Entoderm; ch Chorda dorsalis; $n$ Centralnervensystem; $n$ ' Anlage des peripherischen Nervensystems.

Fig. 13. Ein Querschnitt in der Nähe des Zusammenfließens der beiden Embryonen. Die Bezeichnungen wie auf der Fig. 12. Die ektodermale Brücke $\{e\}$ zwischen den Köpfen der Zwillinge ist schon ganz kurz.

Fig. 14. Ein Querschnitt durch den Anfang des Zusammenfließens der beiden Embryonen Fig. 9, $X I V, 14$ ). Die Bezeichnungen wie anf der Fig. 12. Die Nervenrinnen beider Embryonen stellen die Theile einer gemeinsamen Medullarplatte dar.

Fig. 15. Die Theile der vorhergehenden $(\alpha)$ und der folgenden $(\beta)$ Schnitte verhältnismäßig mit der Fig. 14 bei bedeutender Vergrößerung, $n$ die Wiinde der Nervenrinnen; $n^{\prime}$ der Keim des peripherischen Nervensystems.

Fig. 16. Querschnitt durch die Nackengegend beider Embryonen $A$ und $B$. ¿ das Gewölbe der Medullarplatte zwischen den Medullarrinnen; $n^{\prime}$ der Keim des peripherischen Nervensystems.

Fig. 17. Primitivstreifen ( $p r$ nach 25 stündiger Bebrütung, von 1,75 mm Länge (nach einer mikrophotographischen Aufnahme); ch Kopffortsatz. 
Tafel XV.

Fig. 18. Aus einer Keimsclieibe von 26 Stunden der Bebrütung. Die Länge des Primitivstreifens beträgt $2,5 \mathrm{~mm}$.

Fig. 19. Die Medullarwiilste sind soeben in bedentender Entfernung angelegt. Die Länge des Primitivstreifens $a b$ beträgt $1,7 \mathrm{~mm}$; die des Primitivstreifens $b c \quad 0,5 \mathrm{~mm}$.

Fig. 20. Die Bebrütungszeit ist unbekannt. Die beiden Medullarfalten berühren schon fast die Kopfgegend. Die Kopffalte ist auch vorhanden. Die Länge der oberen Theile des Primitivstreifens $(\alpha b)$ beträgt $0,85 \mathrm{~mm}$; die der hinteren $(b c) 1,5 \mathrm{~mm}$.

Fig. 21. Die Länge des Primitivstreifens bis ztr Zertheilung beträgt $2,15 \mathrm{~mm}$. Die des größeren $Z$ weiges $a b 0,33 \mathrm{~mm}$; die des kleineren ac $0,16 \mathrm{~mm}$.

Fig. 22. Das hintere Ende des Primitivstreifens von 2,65 mm Länge. 